\title{
Clinical Features and Haematological Indices of Neonatal Septicaemia in Poor Resource Setting
}

\author{
Simon Pius' ${ }^{*}$, Mustapha Bello1, Gadzama Bala Galadima², Abdullahi Bukar3, \\ Yakubu Mava ${ }^{4}$, Jose Pwavimbo Ambe ${ }^{1}$ \\ ${ }^{1}$ Dapartment of Paediatrics, University of Maiduguri Teaching Hospital, Maiduguri, Nigeria \\ ${ }^{2}$ Department of Clinical Microbiology, University of Maiduguri Teaching Hospital, Maiduguri, Nigeria \\ ${ }^{3}$ Department of Haematology, University of Maiduguri Teaching Hospital, Maiduguri, Nigeria \\ ${ }^{4}$ Department of Paediatrics, Bingham University Teaching Hospital, Jos, Nigeria \\ Email: "simonpius2000@yahoo.co.uk
}

Received 1 February 2016; accepted 4 March 2016; published 9 March 2016

Copyright (C) 2016 by authors and Scientific Research Publishing Inc.

This work is licensed under the Creative Commons Attribution International License (CC BY). http://creativecommons.org/licenses/by/4.0/

(c) (7) Open Access

\section{Abstract}

Background: The burden of neonatal septicaemia has remained high worldwide and even more severe in the developing countries like ours. Clinical manifestation is variable and non-specific thereby resulting in delay in diagnosis. Blood culture which is the gold standard for diagnosis of neonatal septicaemia (NNS) has many drawbacks due to long waiting time for culture process, low yield, improper inoculation adding to the problem of late diagnosis. Haematological parameters have been utilized in rapid and early diagnosis of NNS and prompt treatment thus circumventing problems associated with drawbacks in blood culture. Objective: The study was to identify the common clinical features of neonatal septicaemia and haematological indices that were commonly utilized in rapid diagnosis of NNS, and also to determine their sensitivity, specificity, positive predictive and negative predictive value. Materials and Methods: The study was prospective and neonates that had clinical features suggestive of neonatal septicaemia were enrolled consecutively into the study. The patients were appropriately investigated including blood cultures, CSF cultures and urine among others, also blood sample for packed cell volume (PCV), total white cell count (TWBC), absolute neutrophil count (ANC), absolute platelet count (APC). Immature to mature neutrophil ratio (I/MNR), immature to total neutrophil ratio (I/TNR) and micro-ESR (erythrocyte sedimentation rate) was also done and analyzed. Results: The common clinical symptoms were fever $\mathbf{3 9 . 5} \%$, poor feeding $\mathbf{3 3 . 6 \%}$, excessive cry $\mathbf{3 8 . 7} \%$, difficulty in breathing $\mathbf{5 0 . 0 \%}$, yellowish skin $26.9 \%$, while the common physical signs were hyper/hypothermia $41.1 \%$, tachypnoea $41.2 \%$, septic umbilical stump $64.0 \%$, hepatomegally $37.3 \%$ and convulsions $42.0 \%$. Blood culture yield was positive in $41.82 \%$ and mortality was as high as $28.00 \%$, the incidence of NNS was 5.9/1000 live births. The haematological parameters as marker of NNS PCV, TWBC, ANC, APC, I/MNR, I/TNR "Corresponding author.

How to cite this paper: Pius, S., Bello, M., Galadima, G.B., Bukar, A., Mava, Y. and Ambe, J.P. (2016) Clinical Features and Haematological Indices of Neonatal Septicaemia in Poor Resource Setting. Open Journal of Pediatrics, 6, 60-68. 
and micro-ESR individually were statistically significant $(P<\mathbf{0 . 0 5})$, also their individual sensitivity, specificity, positive and negative predictive values were highly associated with neonatal septicaemia. However, when they were tested in combinations these markers of neonatal septicaemia had low sensitivity, specificity and their predictive values were weak in excluding NNS. Conclusions: The need for early and rapid diagnosis of NNS is pertinent, culturing of the appropriate specimens remains the only way to identify the aetiological organisms, but is associated with delay. Haematological indices are excellent markers of NNS and analysis is rapid and can easily be done in our laboratory settings, and when utilized efficiently, it would circumvent the delay associated with blood culture for long waiting period for the result, thereby reducing morbidity and mortality.

\section{Keywords}

\section{Neonatal Septicaemia, Clinical Features, Haematological Indices, Early Diagnosis, Poor Resources Setting}

\section{Introduction}

Neonatal septicaemia (NNS) is defined as the clinical syndrome of bacteraemia with symptoms and signs of infection in the first twenty eight days of life [1]. Some authors also define neonatal septicaemia as systemic bacterial infection in a neonate documented by positive blood culture within the first twenty eight days of life [2] [3]. Evaluation of a neonate for possible septicaemia is a challenging clinical condition in newborn intensive care units [2].

Annually, according to World Health Organization (WHO) of the five million death among newborn in Africa and Asia, 1.6 million (20\%) die from neonatal septicaemia [1]. The incidence of neonatal septicaemia varies widely between the developed world and developing countries and also varies from one nursery to another. The characteristic of neonates studied also influences the incidence. For example, the prevalence rate is 3 - 10 fold higher in preterm than in full term neonates [4] [5]. The incidence of neonatal septicemia in developed countries such as Europe and North America is low and ranges between 0.95/1000 live births and 3/1000 live birth [4] [6]. However, in developing countries of Africa and Asia, the incidence is still alarmingly high, ranges from as high as 54.9/1000 live births in Nigeria to around 30/1000 live births in countries like India [7] [8].

Early diagnosis and prompt initiation of antibiotic is desirable, however, newborns with neonatal septicaemia present with vague, non specific symptoms and signs leading to delay in diagnosis and commencement of treatment and resulting in high morbidity and mortality rate. The causes of NNS include intrauterine infection, ascending bacterial infection, and postnatal infection [8]-[10]. Usually clinicians make diagnosis of neonatal septicaemia when the patients present with the following symptoms like fever, poor feeding, excessive cry, respiratory difficulty/grunting, irritability/weakness, vomiting, apnoea, convulsions, skin rashes and unexplained yellowish discoloration on the body [11]. Blood culture is considered as the gold standard for diagnosis of neonatal septicaemia [12]. The drawback associated with blood culture based diagnosis is that it requires high-tech laboratory equipment and well trained laboratory technologist, faulty method of inoculation on culture media and long duration of time of between 36 - 72 hrs of incubation period and up to 10 days before antibiogram sensitivity becomes available, all these lead to delay in prompt intervention [12].

Many attempts has been made to develop a set of screening tests which can rapidly diagnose presence of septicaemia in neonates and prevent a delay associated with waiting for blood culture results in addition to reducing antibiotic wastage in those who are not septicaemic and also can positively correlate with clinical features that are in keeping with neonatal septicaemia. It is suggested that the following simple individual or combinations of haematological parameters such as total white blood cell count (TWBC), absolute neutrophil count (ANC), absolute platelet count (APC), immature to mature neutrophil ratio (I/MNR), immature to total neutrophil ratio (I/TNR), micro-erythrocyte sedimentation rate (Micro-ESR) when analyzed can rapidly diagnose NNS circumventing delay from awaiting blood culture results [1] [13] [14].

A study that looked at clinical features and diagnostic haematological markers of neonatal septicaemia has never been undertaken in our neonatal unit since its inception, we decided to carry out a prospective study that looked at these clinical features and haematological parameters of neonatal septicaemia and explore its use in 
early diagnosis of neonatal septicaemia in a poor resource setting like our neonatal units.

\section{Material and Methods}

This was a consecutive prospective study conducted at the Special Care Baby Unit (SCBU) of Department of Paediatrics, University of Maiduguri Teaching Hospital (UMTH) over a period of twelve months from June 2012 to May 2013. The study was carried on 110 neonates who met the inclusion criteria and detailed history, examination findings were recorded onto study proforma, inclusion criteria were 1) preterm/term neonate with features suggestive of neonatal septicaemia such fever, poor sucking, respiratory distress, 2) Maternal risk factors such as peripartum pyrexia, prolong rupture of membrane and chorio amnionitis, 3) History delivery in unhygienic setting. Exclusion criteria include 1) History of peripartum antibiotic use in the mother or in the neonate prior to presentation 2) Presence of congenital defect in the neonate. 3) History of surgery of surgery prior to presentation and parental decline to consent to included in the research. The patients had blood culture done, and also other sepsis screening was done when it was indicated. Blood for culture was taken on admission from a peripheral vein (antecubital fossa or dorsum of the hand) using a 24 gauge scalp vein needle after thorough skin preparation with application of $2 \%$ iodine tincture and followed by cleansing with $70 \%$ alcohol solution. Two culture bottles, containing $12 \mathrm{mls}$ of culture media- Brain Heart Infusion (BHI) was inoculated aseptically with at least $1 \mathrm{mls}$ of blood each [15] [16] and immediately incubated at $37^{\circ} \mathrm{C}$ for 18 - $24 \mathrm{hrs}$. Each specimen was examined twice a day for growth. Turbidity in the culture bottles and/or excess production of gas with signs of haemolysis indicates bacterial growth. It was sub-cultured on blood agar using sheep blood and/or MacConkey agar and this was incubated at $37^{\circ} \mathrm{C}$ for $18-24 \mathrm{hrs}$. Other specimen that was cultured includes urine, abscesses and cerebrospinal fluid when it was indicated. When the culture was positive sensitivity pattern was carried out against the common antibiotic discs provided by the use of disc diffusion method according to clinical and laboratory standard institute (CLSI) [16].

Another $2 \mathrm{ml}$ of blood was also collected in an EDTA container for a complete haematological profile by direct counting using an improved Neubaur's Chamber. The packed cell volume (PCV), total white blood cell count (TWBC), with differential white cell count, erythrocyte sedimentation rate (Micro-ESR) was also estimated, leucocytes morphology and absolute neutrophil count, absolute platelet count (APC) as well as immature to mature neutrophil ratio (I/MNR), immature to total neutrophil ratio (I/TNR) was estimated on peripheral blood smear (PBS) stained with Leishmann's stain and value $>0.2$ was considered abnormal [1] [14].

The recorded data was analyzed using statistical package for social sciences (SPSS) version 16.0 (SPSS software Inc, Chicago, USA). Tables were used for data presentation as appropriate. Associations were tested using Chi-square and student-t test where appropriate, while measures of means and standard deviation of continuous variables were computed. Statistical significance was set at $\mathrm{p}<0.05$.

\section{Results}

One hundred and ten neonates met the inclusion criteria and were enrolled into the study. Of these, $66.00 \%$ were male while $34.00 \%$ were female, giving male to female ratio of $1.9: 1$. Inborn constituted $38.18 \%$ while $61.82 \%$ were outborn, the mean age and weight at admission were $5.33 \pm 5.29$ days and $2842.27 \pm 734.23$ g respectively. Preterm neonates constituted $16.36 \%$ of the enrolled neonates. Of the newborn $41.80 \%$ had blood culture positive results for neonatal septicaemia which give the incidence of neonatal septicaemia at 5.9/1000 live births.

There were equal representation of Gram positive and Gram negative organism cultured. Of the Gram positive, Staphylococcus aureus was predominant with $70.00 \%$, followed by Streptococcus pyogenes (22.00\%). Among the Gram negative bacteria, Escherichia coli was predominant constituting 39.10\%, followed by Klebsiella pneumoniae (30.50\%) and Coliform spp with (21.80\%). Mortality rate was $28.00 \%$, and mortality was higher among those with Gram negative organism septicaemia than those due to Gram Positive organisms.

\section{Clinical Symptoms of Neonatal Septicaemia}

Table 1 shows the frequency of symptoms among culture proven in 110 neonates studied. The commonest symptoms of admitted patients identified were fever, poor feeding, and excessive crying. Respiratory grunting and diarrhoea were the least common symptoms. Specifically, fever, poor feeding and irritability were the most common symptoms in babies with culture proven septicaemia see Table 1. 
Table 2 shows the physical signs among culture proven in the 110 neonates studied. Hyperthermia/hypothermia and tachypnoea were frequent physical findings. Of those with hyperthermia, rectal (core) temperature measurement identified more neonates with septicaemia more than the axillary temperature recording. Out of 95 neonates with core (rectal) temperature $>37.5^{\circ} \mathrm{C}, 39(41.1 \%)$, had positive blood culture. Ten had normal temperature out of which, 3 (30.0\%) had positive blood culture. There were 5 neonates with hypothermia $\left(\mathrm{t}<36.5^{\circ} \mathrm{C}\right)$

Table 1. Frequency of symptoms in relation to 46 neonates with positive blood cultures.

\begin{tabular}{ccc}
\hline Clinical symptoms & Number of neonates & Number of neonates with septicaemia \\
Fever & 96 & $39(40.6)$ \\
Poor feeding & 70 & $33(47.1)$ \\
Excessive cry & 36 & $14(38.9)$ \\
Difficulty in breathing & 26 & $13(50.0)$ \\
Jaundice & 26 & $7(26.9)$ \\
Irritability & 25 & $16(64.0)^{*}$ \\
Skin rashes & 16 & $9(56.3)$ \\
Jitteriness & 16 & $16(31.2)$ \\
Spasms & 12 & $4(33.3)$ \\
Lethargy & 10 & $8(80.0)^{*}$ \\
Grunting respiration & 5 & $1(20.0)$ \\
Diarrhoea & 4 & $2(50.0)$ \\
\hline
\end{tabular}

"Statistical significance set $\mathrm{p}<0.05$.

Table 2. Physisal signs in relation to 46 neonates with septicaemia.

\begin{tabular}{|c|c|c|}
\hline Physical signs & Total number of neonates & No. of neonates with culture proven septicaemia (\%) \\
\hline \multicolumn{3}{|l|}{ Rectal temperature $\left({ }^{\circ} \mathrm{C}\right)$} \\
\hline Hyperthermia $(\geq 37.6)$ & 95 & 39 (41.1) \\
\hline Normal (36.5 - 37.5) & 10 & $3(30.0)$ \\
\hline Hypothermia $(<36.5)$ & 5 & $4(80.0)$ \\
\hline \multicolumn{3}{|l|}{ Axillary temperature $\left({ }^{\circ} \mathrm{C}\right)$} \\
\hline Hyperthermia ( $\geq 37.6$ ) & 77 & $32(41.6)$ \\
\hline Normal (36.5 - 37.5) & 26 & $9(34.6)$ \\
\hline Hypothermia $(<36.5)$ & 7 & $5(71.4)$ \\
\hline Tachypnoea & 34 & $14(41.2)$ \\
\hline Septic umbilicus & 11 & $7(64.0)$ \\
\hline Hepatomegally & 8 & $3(37.5)$ \\
\hline Tachycardia & 7 & $4(57.1)$ \\
\hline Convulsion & 7 & $3(42.8)$ \\
\hline Intercostal recession & 5 & $3(60.0)$ \\
\hline Hyper/hypothermia & 5 & $2(40.0)$ \\
\hline Scalding & 4 & $2(50.0)$ \\
\hline Apnoea & 3 & $1(33.3)$ \\
\hline Abdominal distension & 2 & $2(100.0)$ \\
\hline Joint swelling & 2 & $1(50.0)$ \\
\hline Sclerema & 1 & $0(0.0)$ \\
\hline Crepitation & 1 & $0(0.0)$ \\
\hline Murmur & 1 & $0(0.0)$ \\
\hline
\end{tabular}


out of which, 4 (80.0\%) had positive blood culture. There was no statistically significant association $\left(\chi^{2}=0.441\right.$, $\mathrm{p}=0.506)$ Based on axillary temperature recording, 77 neonates with hyperthermia out of which $32(41.6 \%)$ had positive blood culture. There were 26 (23.6\%) neonate with normal temperature recording out of which 9 (34.6\%) had positive blood culture. Seven (6.4\%) neonates had hypothermia 5 (71.4\%) had positive blood culture. There was no statistically significant difference $\left(\chi^{2}=0.441, \mathrm{p}=0.506\right)$. Other physical sign are presented in table below and they are not statistically significant $(\mathrm{p}=0.05)$.

Table 3 shows selected haematologic indices among neonates with positive and negative blood culture. Packed cell volume (44.50\%), lymphocytes (26.67\%) and absolute platelets count $\left(148.90 \times 10^{9} / \mathrm{L}\right)$ were significantly low in septicaemic neonates. Also neutrophils (70.48\%), micro-ESR $(9.47 \mathrm{~mm} / \mathrm{hr})$, the ration of immature to mature neutrophils $(0.29)$, as well as immature to total neutrophils $(0.26)$ were significantly higher in septicaemic babies.

In Table 4, the TWBC showed sensitivity of $46 \%$, its specificity was $51 \%$, while the positive and negative predictive values were $40 \%$ and $57 \%$ respectively. The ANC had sensitivity of $96 \%$, specificity of $25 \%$, its positive predictive value was $89 \%$ and the negative predictive value was $48 \%$. Absolute platelet count showed sensitivity of $90 \%$ and specificity of $56 \%$. It had positive predictive value of $81 \%$ and the negative predictive value of $71 \%$. Immature to total neutrophil ratio showed sensitivity of $83 \%$ and specificity of $58 \%$, while the positive predictive value was $75 \%$ and the negative predictive value was $74 \%$. Immature to mature neutrophil ratio had sensitivity of $80 \%$ and specificity of $75 \%$, while the positive predictive value was $85 \%$ and the negative predictive value was $88 \%$. Micro-ESR had sensitivity of $80 \%$ and specificity of $51 \%$ while the positive predictive value was $31 \%$ and the negative predictive value was $25 \%$. All the values are summarized in Table 4.

In Table 5, the results of combination of the parameters are as shown, TWBC, ANC and APC had sensitivity of $66 \%$, specificity of $55 \%$, positive predictive value of $56 \%$ and negative predictive value of $72 \%$. Four combinations of TWBC, ANC, APC and Micro-ESR had a sensitivity of 70\%, specificity of 54\%, positive predictive value of $50 \%$ and negative predictive value of $72 \%$. When all the six parameters were combined the sensitivity

Table 3. Shows hematological parameters in neonatal septicaemia.

\begin{tabular}{|c|c|c|c|c|}
\hline \multirow{2}{*}{ Haematological parameters (unit) } & \multicolumn{2}{|c|}{ Blood culture Mean (SD) } & \multirow{2}{*}{$\mathrm{t}$-Test } & \multirow{2}{*}{$\mathrm{p}$ - value } \\
\hline & Positive (46) & Negative (64) & & \\
\hline PCV (\%) & $44.50(8.08)$ & $47.86(6.54)$ & 2.326 & $0.022^{*}$ \\
\hline TWBC $\left(10^{9} / \mathrm{L}\right)$ & $9.41(2.40)$ & $8.57(3.08)$ & 1.545 & 0.125 \\
\hline Neutrophil (\%) & $70.48(6.24)$ & $65.84(6.92)$ & 3.609 & $0.000^{*}$ \\
\hline Lymphocyte (\%) & $26.67(6.60)$ & $29.84(6.75)$ & 2.453 & $0.016^{*}$ \\
\hline Micro-ESR (mm/hr) & $9.43(4.74)$ & $5.70(3.23)$ & 4.882 & $0.000^{*}$ \\
\hline Absolute platelet count $\left(10^{9} / \mathrm{L}\right)$ & $148.90(34.55)$ & $196.8(44.46)$ & 6.044 & $0.000^{*}$ \\
\hline Immature to mature Neutrophil & 0.29 (11.39) & $0.17(0.08)$ & 6.631 & $0.000^{*}$ \\
\hline Immature to total Neutrophil & $0.26(0.05)$ & $0.15(0.08)$ & 7.562 & $0.000^{*}$ \\
\hline
\end{tabular}

* Statistical significance is taken at $\mathrm{p}<0.05$.

Table 4. Sensitivity, specificity and predictive values of individual haematological parameters in culture proven neonatal septicaemia.

\begin{tabular}{|c|c|c|c|c|}
\hline Tests & Sensitivity (\%) & Specificity (\%) & PPV (\%) & NPV (\%) \\
\hline TWBC $\left(<5000\right.$ or $\left.>18,000 / \mathrm{mm}^{3}\right)$ & 46 & 51 & 40 & 57 \\
\hline ANC $\left(>6000\right.$ or $\left.<1500 / \mathrm{mm}^{3}\right)$ & 96 & 25 & 89 & 48 \\
\hline $\operatorname{APC}\left(<150 / \mathrm{mm}^{3}\right)$ & 90 & 56 & 81 & 71 \\
\hline I/TNR (>0.25) & 83 & 58 & 75 & 74 \\
\hline $\mathrm{I} / \mathrm{MNR}(>0.26)$ & 80 & 75 & 85 & 88 \\
\hline Micro-ESR (>15 mm/hr) ${ }^{*}$ & 80 & 51 & 31 & 25 \\
\hline
\end{tabular}

* sedimentation in the first hour, PPV = positive predictive value, NPV = negative predictive value. 
Table 5. Combination of haematological indices tests in culture proven neonatal septicaemia.

\begin{tabular}{ccccc}
\hline Tests & Sensitivity (\%) & Specificity (\%) & PPV (\%) & NPV (\%) \\
\hline TWBC + ANC + APC & 66 & 55 & 56 & 72 \\
TWBC + ANC + APC + Micro-ESR & 70 & 54 & 50 & 60 \\
TWBC + ANC + APC + Micro-ESR + I/TNR + I/MNR & 69 & 63 & 60 & 67 \\
\hline
\end{tabular}

$\mathrm{PPV}=$ positive predictive value, $\mathrm{NPV}=$ negative predictive value.

was $69 \%$, specificity was $63 \%$, and positive predictive value of $60 \%$ and negative predictive of $67 \%$ as summarized in Table 5.

\section{Discussion}

Neonatal septicaemia has remained a burden all over the world and is more severe in the developing countries. This study showed a high mortality and high complication among survivors, this was similar to the assertion by Arif and co-workers [1]. The need for early recognition, diagnosis of neonatal septicaemia and prompt institution of treatment is paramount so as to prevent unwanted death and complication associated with neonatal septicaemia, but it is quiet often difficult and delayed because of the variable and non-specific clinical manifestation of neonatal septicaemia [14]. Some of the clinical features that are presumably ascribed to neonatal infections can also occur in other neonatal conditions like hypoglycaemia, perinatal asphyxia among others [11] [12] [14].

The clinical symptoms were fever, poor feeding, excessive cry, difficulty in breathing, yellowish skin discoloration, skin rashes, jitteriness and irritability; they were the common features at admission in the study subjects. Hyperthermia, hypothermia, tachypnoea, septic umbilical stump hepatomegally, skin lesion and convulsions were the common physical signs observed, this was similar to those that were reported by some workers [1] [14] [17]. It was also observed that features like weakness, hypothermia and apnoea were more common among preterm septic neonates, while excessive cry, pyrexia and convulsions were more in the term septicaemic neonates and these observation was similar to the finding in Asia and America [1] [10] [17].

Blood culture which is the gold standard for diagnosis of neonatal septicaemia was positive in $41.20 \%$ that means 58.8\% of the presumed septicaemic patients could have been excluded. Arif et al. [1] and Sharma et al. [18] had reported fatal infections in the presence of negative blood culture. The reason for such a negative results could be due to intermittent or low-density bacteremia. Other reason could be due to intrapartum antibiotic administration thus suppressing growth [1] [19]. Some studies have also documented blood culture to be positive in $30 \%$, and $42 \%$ of cases of NNS [1], and these agrees with our result of $41.20 \%$. It means that more than half of the cases of neonatal septicaemia could have been missed if diagnosis was only based on positive blood culture [1] [18] [20].

This study evaluated these simple and common haematological indices that can be done in laboratory of most developing countries like ours, it was found that these parameters had a good correlation with blood culture positivity and was statistically significant when it was subjected to Student-T tests $(\mathrm{p}<0.05)$. Such an observation was also reported by Monroe et al. [21], and Ottolini et al. [22]. However, it was not so by other workers who did not find any relationship between haematological indices and blood culture positive neonatal septicaemia [23]. In the this study, TWBC $<5000$ or $>18,000$ had low sensitivity, specificity, positive and predictive value in determining neonatal septicaemia and this was in agreement with the report from Pakistan [4], but disagree with report by Schelonka and co-workers [24] who observed a higher values in these range.

Absolute neutrophil count (ANC) had high sensitivity and positive predictive value but low specificity and low negative predictive value; this was corroborated by the report by Arif and co-workers [1]. Absolute platelet counts (APC) had high sensitivity $90 \%$, high positive predictive value $81 \%$, but low specificity $56 \%$, while immature to mature neutrophil ratio (I/MNR), sensitivity of $80 \%$, specificity of $75 \%$, positive predictive value of $85 \%$ and negative predictive value of $88 \%$, similar finding has been reported by Buch and co-workers [14], but at variance with earlier report by Gerdes who reported low specificity and low negative predictive values [25].

In the current study the immature to total neutrophil ratio's (I/TNR) high sensitivity was in concordance with earlier report [14] [24]. Micro-ESR had high sensitivity rate findings is in concordance with similar report from Pakistan and India [1] [18] [25]. The combination of three TWBC, ANC and APC had weak sensitivity, specificity and positive predictive value but had good negative predictive value. 
Also when six parameters were combined that is TWBC, ANC, and APC, micro-ESR, I/MNR and I/TNR their sensitivity, specificity, positive predictive and negative predictive values were low in blood culture neonatal septicaemia and had even weaker correlation with clinical features among these septicaemic neonates. This finding corroborated well with findings by NNPD [7], Varsha and co-workers [26], but disagree with work by Buch who reported higher sensitivity, higher positive predictive values and negative predictive values [14].

\section{Conclusions}

In this study we affirm that the burden of neonatal septicaemia has remained high in our setting and the clinical presentations; symptoms and signs are protean and non-specific, leading to delay in diagnosis. NNS is also associated with high mortality and morbidity among survivors; thus there is an urgent need for early diagnosis and prompt institution of antibiotics.

There is no doubt that blood culture based diagnosis has remained the only way to identify the causative organisms, but it requires well equipped laboratory and trained personnel that are lacking in most of our setting, it also takes long time and NNS has rapid course and the fatality rate is high (mortality rate $28.0 \%$ ).

In order to circumvent the problems of delay and low yield associated with blood culture based diagnosis, a set of simple, rapid and easily processed haematological markers of NNS such as PCV, TWBC, ANC, APC, I/ MNR, I/TNR and Micro-ESR had significant values in making early diagnosis of presence of NNS and to certain degree exclude NNS. The strength of their sensitivity, specificity, positive predictive values and negative predictive values of the individually parameters was good. Since these haematological parameters are less cumbersome and processed early enough, it can be utilized in the early diagnosis of NNS in our settings.

\section{Limitation}

Due to the high cost of financing of the research work, we could not afford to repeat the blood culture which would have been desirable in order to improve the bacterial yield. Also in the same vein we could not afford to study the C reactive protein (C-RP), interleukine-6 (IL-6) and interleukine-8 (IL-8) which are currently used individually or in combination as set of screening tests tools for the purpose of early diagnosis of neonatal septicaemia and it could have possibly make our findings more plausible.

\section{Acknowledgements}

The authors are particularly grateful to the staff of the Special Care Baby Unit and the Medical Record Department of UMTH for their support during the study.

\section{Funding}

This research was funded by the authors.

\section{Ethical Approval}

Ethical approval was obtained from the Hospital Research and Ethics Committee.

\section{Conflict of Interest}

None declared.

\section{Contributors}

Pius S conceived the research and with other authors collected and analyzed the data, wrote the draft and critical reviewed the final article.

\section{References}

[1] Arif, S., Ehsen, A., Arif, M., Hussein, J. and Bano, R. (2013) Early Diagnosis of Neonatal Sepsis through Haematological and Biochemical Markers. Gomal Journal of Medical Sciences, 11, 178-182.

[2] Elegba, O.Y., Babaniyi, I.B. and Iregbu, K.C. (2006) Bacteriological Profile of Neonatal Septicaemia in a Tertiary 
Hospital in Nigeria. African Health Sciences, 6, 151-154.

[3] Ojukwu, J.U., Abonyi, L.E. and Orji, I.K. (2005) Neonatal Septicaemia in High Risk Babies in South Eastern Nigeria. Journal of Perinatal Medicine, 34, 166-172.

[4] Ahmed, Z., Ghafoor, T., Waqar, T., Ali, S., Aziz, S. and Mahmud, S. (2005) Diagnostic Value of C-Reactive Protein and Haematological Parameters in Neonatal Sepsis. Journal of the College of Physicians and Surgeons Pakistan, 15, 152-156.

[5] Alausa, O.K. and Montefiore, D. (1978) Bacterial Infection, Sensitivity Patterns and Chemotherapy among Hospital Patients in the Tropics. Scandinavian Journal of Infectious Diseases, 10, 295-302. http://dx.doi.org/10.3109/inf.1978.10.issue-4.07

[6] Vesikeri, T., Janas, M., Gronroos, P., Tuppurainen, N., Renland, M., Kero, P., et al. (1985) Neonatal Septicaemia. Archives of Disease in Childhood, 60, 542-546. http://dx.doi.org/10.1136/adc.60.6.542

[7] Department of Paediatrics (No Date) National Neonatal Perinatal Database (2002-2003) (Online). All India Institute of Medical Sciences, NNPD Nodal Center, New Delhi. http://www.newbornwhocc.org/pdf/HRRC_Report_2002-03.pdf

[8] Anah, M.U., Udo, J.J., Ochigbo, S.O. and Abia-Bassey, L.N. (2008) Neonatal Septicaemia in Calabar, Nigeria. Tropical Doctor, 38, 126-128. http://dx.doi.org/10.1258/td.2006.006037

[9] Yoon, B.H., Romero, R., Moon, J.B., et al. (2001) Clinical Significance of Intramniotic Inflammation in Patients with Preterm Labour and Intact Membranes. American Journal of Obstetrics and Gynecology, 185, 1130-1136. http://dx.doi.org/10.1067/mob.2001.117680

[10] Sohn, A.H., Garret, D.O., Sinkowitz, R.L., et al. (2001) Prevalence of Nosocomial Infections in Neonatal Intensive Care Unit Patients. Journal of Pediatrics, 139, 821-827. http://dx.doi.org/10.1067/mpd.2001.119442

[11] Robertson, C.M. and Coopersmith, C.M. (2006) The Systemic Inflammatory Response Syndrome. Microbes and Infection, 8, 1382-1389. http://dx.doi.org/10.1016/j.micinf.2005.12.016

[12] Haque, K.N. (2010) Neonatal Sepsis in Very Low Birth Weight Preterm Infants, Part 2: Review of Definition, Diagnosis and Management. Journal of Medical Sciences, 3, 11-27.

[13] Levy, M.M., Fink, M.P., Marshall, J.C., Abraham, E., Angus, D., Cook, D., et al. (2003) International Sepsis Definition Conference. Critical Care Medicine, 31, 1250-1256. http://dx.doi.org/10.1097/01.CCM.0000050454.01978.3B

[14] Buch, A.C., Srivastava, V., Kumar, H. and Jadhav, P.S. (2011) Evaluation of Haematological Profile in Early Diagnosis of Clinically Suspected Cases of Neonatal Sepsis. International Journal of Basic and Applied Medical Sciences, 1, $1-6$.

[15] Cheesbrough, M. (2000) Microbiological Tests. In: Cheesbrough, M., Ed., District Laboratory Practice in Tropical Countries, Part II, Low Priced Edition, Cambridge University Press, Cambridge, 105-130.

[16] CLSI (2011) Performance Standards for Antimicrobial Susceptibility Testing: Twenty-First Informational Supplement. CLSI Document M100-S21. Clinical and Laboratory Standard Institute, Wayne, Pennsylvania.

[17] Waseem, R., Khan, M., Tahira, S.I. and Waheed, A. (2005) Neonatal Sepsis. Professional Medical Journal, 4, 451456.

[18] Sharma, A., Krishna-Kutty, C.V., Sabharwal, U. and Sushila-Mohan, H. (1993) Evaluation of Sepsis Screen for Diagnosis of Neonatal Septicaemia. Indian Journal of Pediatrics, 60, 559-563. http://dx.doi.org/10.1007/BF02751434

[19] Pourcyrous, M, Bada, H.S., Korones, S.B., Baselski, V. and Wong, S.P. (1993) Significance of C-Reactive Protein Responses in Neonatal Bacterial Infection and Other Disorders. Pediatrics, 92, 431-435.

[20] Berger, C., Uehlinger, J., Ghelfi, D., Blau, N. and Fanconi, S. (2005) Comparison of C-Reactive Protein and White Blood Cell Count with Differential in Neonates at Risk for Septicaemia. European Journal of Pediatrics, 154, 139-144.

[21] Monroe, B.L., Weiberg, A.G., and Rosenfield, C.R. (1979) Neonatal Blood Count in Health and in Disease. I. Reference Values for Neutrophilic Cells. Journal of Pediatrics, 95, 89-98. http://dx.doi.org/10.1016/S0022-3476(79)80096-7

[22] Ottolini, M.C., Lundgreen, K. and Mirkinson, L.J. (2003) Utility of Complete Blood Count and Blood Culture Screening to Diagnose Neonatal Sepsis in the Asymptomatic at Risk Newborn. The Pediatric Infectious Disease Journal, 22, 430-434. http://dx.doi.org/10.1097/01.inf.0000068206.11303.dd

[23] Rosycki, H.J., Stahl, G.E. and Baumgart, S. (1987) Impaired Sensitivity of a Single Early Leucocyte Count in Screening for Neonatal Sepsis. The Pediatric Infectious Disease Journal, 6, 440-442. http://dx.doi.org/10.1097/00006454-198705000-00004

[24] Schelonka, R.L., Bradley, Y.A. and Desjardins, S.E. (1994) Peripheral Leucocyte Count and Leucocyte Indexes in Healthy Term Newborn Infants. Journal of Pediatrics, 125, 603-606. http://dx.doi.org/10.1016/S0022-3476(94)70018-4 
[25] Gerdes, J.S. and Polin, R. (1998) Early Diagnosis and Treatment of Neonatal Sepsis. Indian Journal of Pediatrics, 65, 63-78. http://dx.doi.org/10.1007/BF02849696

[26] Varsha, S., Rusia, U., Shikka, M., Faridi, M.M.A. and Madan, M. (2003) Validity of Haematological Parameters in Identification of Early and Late Onset Neonatal Infection. Journal of Paediatrics and Child Health, 46, 565-568. 